\title{
Quasielastic Light Scattering from Polydisperse Polymer Solution
}

\author{
Tsuruyo TANAKA \\ Department of Material Science, Faculty of Science, \\ Hiroshima University, Hiroshima Japan.
}

(Received May 31, 1974)

\begin{abstract}
A new theory is proposed to investigate the effect of polydispersity on the quasielastic light scattering of polymer solutions. Spectral distribution and autocorrelation function of the field of the light scattered from a polydisperse solution are investigated. The autocorrelation function of the photocurrent is expressed in terms of a Laplace transform of a suitably defined molecular-weight distribution function. Average diffusion constant of a polydisperse system is obtained directly from the autocorrelation function. It gives the exact value, and hence is superior to the conventional method which uses a Lorentzian approximation for the spectral distribution.

KEY WORDS Light Scattering / Polydisperse Polymer / Diffusion

Constant / Autocorrelation Function /
\end{abstract}

The conventional study of quasielastic light scattering of polymer solution is based on the analysis of the spectral distribution which is a Fourier transform of the time-autocorrelation function of the field of the light scattered from the solution. The recent development of technology has made possible a direct measurement of the autocorrelation function. This approach is much simpler and more rigorous for a polydisperse solution than the spectral treatment. The author attempts to obtain average diffusion constant of a polydisperse polymer solution theoretically from the autocorrelation function.

For a monodisperse system, a general theory of the spectral distribution of the Rayleigh line has been developed by Pecora. ${ }^{1-7} \mathrm{He}$ found that the spectrum is given by a Lorentzian distribution and that the translational diffusion constant of the system is obtained from the halfwidth at the half-maximum of the spectrum. Cummins, et al., ${ }^{8}$ has developed a phenomenological theory of the autocorrelation function using the relation between the macroscopic diffusion constant and a statistical average of the molecular motion. Although their conclusions are equivalent to those of Pecora, their theory is useful for the analysis of experimental data for the quasielastic light scattering.

The effect of polydispersity on the spectral distribution has been treated by several authors. ${ }^{9-12}$ Pecora and Tagami ${ }^{9}$ have calculated the spectrum of a polydisperse system using Schulz-Zimm's distribution of the molecular weight and have shown that the average diffusion constant of a polydisperse rigid-rod solution obtained from the half-width of the spectrum gives a value lower than that of a monodisperse system with the same mean molecular weight. Koppel ${ }^{12}$ has recently proposed a formulation by means of the method of cummulant analysis of the spectrum of the polydisperse system. Since the spectrum of the polydisperse system deviates from a Lorentzian distribution, the average diffusion constant obtained from the half-width of the spectrum does not give an exact value. In the case of a polydisperse system, it is more appropriate to treat the autocorrelation function directly.

There has been a little amount of information available about the effect of polydispersity on the autocorrelation function. In this paper, the autocorrelation function formulation developed by Cummins, et al., is extended to the polydisperse system assuming the additivity of the fields scattered from different scatterers. An apparent molecular-weight distribution function is introduced, the Laplace transform of which gives the autocorrelation function of the photocurrent. The spectral distribution of a polydisperse system is also derived. The average diffusion constant of a polydisperse system is obtained directly from the autocorrelation function without assuming a 
particular distribution for the spectrum. The characteristic properties of the average diffusion constant of the polydisperse system are discussed. Average diffusion constants of several model systems are numerically calculated both from the autocorrelation function and from the spectrum. The utility of the autocorrelation function formulation is discussed by comparing it with the conventional theory.

\section{EFFECT OF THE MOLECULAR-WEIGHT DISTRIBUTION ON QUASIELASTIC LIGHT SCATTERING}

It is shown by Cummins, et al., that the autocorrelation function of the scattered field from spherical scatterers of the same size undergoing translational diffusion is written as

$$
\begin{aligned}
C_{E}(\tau) & =\left\langle E^{*}(t) E(t+\tau)\right\rangle \\
& =N|A|^{2} \exp \left(-i \omega_{0} \tau\right) \exp \left(-D_{\mathrm{T}} k^{2} \tau\right)
\end{aligned}
$$

In eq. $1, N$ is the number of scatterers, $|A|$ is the magnitude of the scattering amplitude, $\omega_{0}$ is the frequency of the incident light, $D_{\mathrm{T}}$ is the translational diffusion constant and

$$
k=\frac{4 \pi}{\lambda} \sin \frac{\theta}{2}
$$

where $\lambda$ is the wave length of the incident light and $\theta$ is the scattering angle. It is assumed that scatterers are uncorrelated to each other. Using Wiener-Khinchine theorem, the spectrum has a following Lorentzian distribution as has been shown by several authors:

$$
\begin{aligned}
I(\omega) & =\frac{1}{2 \pi} \int_{-\infty}^{\infty} C_{\mathrm{E}}(\tau) \exp (-i \omega \tau) \mathrm{d} \tau \\
& =\frac{N|A|^{2}}{\pi} \frac{D_{\mathrm{T}} k^{2}}{\left(\omega-\omega_{0}\right)^{2}+\left(D_{\mathrm{T}} k^{2}\right)^{2}}
\end{aligned}
$$

The diffusion constant of the solution is obtained from the half-width at the half-maximum of the Lorentzian spectrum:

$$
\Delta \omega_{1 / 2}=D_{\mathrm{T}} k^{2}
$$

For a polydisperse polymer solution, we assume the additivity of the fields scattered from different molecules. The autocorrelation function of the scattered field is then given by

$$
C_{\mathrm{E}}(\tau)=\Sigma_{i} N_{i}|A|_{i}{ }^{2} \exp \left(-i \omega_{0} \tau\right) \exp \left(-D_{\mathrm{T} i} k^{2} \tau\right)
$$

where the suffix $i$ refers to the $i$-th species of molecule. Equation 5 is conveniently written using a continuous distribution of the molecular weight as

$$
\begin{aligned}
C_{\mathrm{E}}(\tau)= & \int_{0}^{\infty} f(M)|A(M, \theta)|^{2} \exp \left(-i \omega_{0} \tau\right) \\
& \times \exp \left(-D_{\mathrm{T}}(M) k^{2} \tau\right) \mathrm{d} M
\end{aligned}
$$

where $f(M) \mathrm{d} M$ is the weight fraction of the molecules with molecular weight between $M$ and $M+\mathrm{d} M$. We obtain the spectral distribution in the form

$$
I(\omega)=\frac{1}{\pi} \int_{0}^{\infty} \frac{D_{\mathrm{T}}(M) k^{2} f(M)|A(M, \theta)|^{2}}{\left(\omega-\omega_{0}\right)^{2}+\left(D_{\mathrm{T}}(M) k^{2}\right)^{2}} \mathrm{~d} M
$$

Equation 7 is not necessarily a Lorentzian distribution in contrast to the monodisperse system.

The photoelectric current is proportional to the probability that a photoelectron will be emitted, and is defined as

$$
i(t)=e \beta E^{*}(t) E(t)
$$

where $\beta$ is a suitably defined quantum efficiency. It was also shown by Cummins, et al., that the autocorrelation function of the photocurrent from a monodisperse system is given by

$$
\begin{aligned}
C_{i}(\tau) & =\langle i(t) i(t+\tau)\rangle \\
& =e\langle i\rangle \delta(\tau)+\langle i\rangle^{2}+\langle i\rangle^{2}\left(\frac{N|A|^{2} \exp \left(-D_{\mathrm{T}} k^{2} \tau\right)}{N|A|^{2}}\right)^{2}
\end{aligned}
$$

where $\langle i\rangle$ is the average photocurrent. This equation is based on the theory that the secondorder correlation function is expressed by the first-order correlation function for a Gaussian fluctuation. In eq 9, the first term is the shot noise and the second a D.C. signal which can be blocked in a real experiment. We shall be interested only in the last term of eq 9 and denote it by $C^{\prime}(\tau)$ :

$$
C^{\prime}(\tau)=\langle i\rangle^{2}\left[\frac{N|A|^{2} \exp \left(-D_{\mathrm{T}} k^{2} \tau\right)}{N|A|^{2}}\right]^{2}
$$

We extend eq 10 to a polydisperse system in a way similar to eq 5 , obtaining

$$
C^{\prime}(\tau)=\langle i\rangle^{2} \frac{\Sigma_{i} N_{i}\left|A_{i}\right|^{2} \exp \left(-D_{\mathrm{T} i} k^{2} \tau\right)}{\sum_{i} N_{i}\left|A_{i}\right|^{2}}
$$

Eq 11 is again expressed by the weight distribution function of the molecular weight $f(M)$ as 


$$
C^{\prime}(\tau)=\langle i\rangle^{2}\left[\frac{\int_{0}^{\infty} f(M)|A(M, \theta)|^{2} \exp \left(-D_{\mathrm{T}}(M) k^{2} \tau\right) \mathrm{d} M}{\int_{0}^{\infty} f(M)|A(M, \theta)|^{2} \mathrm{~d} M}\right]^{2}
$$

We denote $\left[C^{\prime}(\tau)\right]^{1 / 2} /\langle i\rangle$ by $C(\tau)$ and obtain

$$
C(\tau)=\frac{\int_{0}^{\infty} f(M)|A(M, \theta)|^{2} \exp \left(-D_{\mathrm{T}}(M) k^{2} \tau\right) \mathrm{d} M}{\int_{0}^{\infty} f(M)|A(M, \theta)|^{2} \mathrm{~d} M}
$$

We define the apparent molecular-weight distribution function $W(M, \theta)$ as follows:

$$
W(M, \theta)=\frac{f(M)|A(M, \theta)|^{2}}{\int_{0}^{\infty} f(M)|A(M, \theta)|^{2} \mathrm{~d} M}
$$

Note that $W(M, \theta)$ depends on the scattering angle. Substituting eq 14 into eq 13 , we get

$$
C(\tau)=\int_{0}^{\infty} W(M) \exp \left(-D_{\mathrm{T}}(M) k^{2} \tau\right) \mathrm{d} M
$$

This is the basic expression for the autocorrelation function of the photocurrent for a polydisperse system.

The dependence of $W(M, \theta)$ on the scattering amplitude $|A|$ is important in the case of the polydisperse system in contrast to the case of a monodisperse system. In particular, when the size of the scatterers becomes comparable to or larger than the wave length of the incident light, the scattering amplitude, namely, the intensity of the light scattered in some forward angle is different from that scattered at its supplementary angle. ${ }^{13}$ This effect becomes remarkable as the size of the particle increases. In addition, the contribution from larger particles to the scattering amplitude is larger than that from smaller particles.

By the transformation $x=D_{\mathrm{T}}(M) k^{2}$, eq 15 can be put in the form of a Laplace transform, i.e.,

$$
C(\tau)=\int_{0}^{\infty} W(M) \exp (-x \tau) \frac{1}{k^{2}}\left[\frac{\mathrm{d} D_{\mathrm{T}}(M)}{\mathrm{d} M}\right]^{-1} \mathrm{~d} x
$$

The inversion formula of the Laplace transform is well known: namely, if

$$
F(t)=\int_{0}^{\infty} G(x) \exp (-x t) \mathrm{d} x
$$

then

$$
G\left(x=t^{-1}\right)=\lim _{\nu \rightarrow \infty}\left[\frac{(-1)^{\nu}}{\nu !}\right](\nu t)^{\nu+1}\left[\frac{\mathrm{d}^{\nu}}{\mathrm{d} t^{\nu}} F(\nu t)\right]
$$

Application of this inversion foumula to eq 16 gives for the apparent molecular weight distribution function the expression

$$
\begin{aligned}
W(M, \theta)= & k^{2}\left[\frac{\mathrm{d} D_{\mathrm{T}}(M)}{\mathrm{d} M}\right] \\
& \times \lim _{\nu \rightarrow \infty}\left[\frac{(-1)^{\nu}}{\nu !}\right](\nu t)^{\nu+1} \frac{\mathrm{d}^{\nu}}{\mathrm{d} t^{\nu}} C(\nu \tau)
\end{aligned}
$$

This result can be used to calculate the molecularweight distribution function of the polydisperse system from the data on the autocorrelation function of the photocurrent if the molecular weight dependence of $D_{\mathrm{T}}(M)$ is known.

The normalized intensity is usually defined as

$$
P(\theta)=\frac{|A(M, \theta)|^{2}}{|A(M, 0)|^{2}}
$$

and $P(\theta)$ is called the particle scattering factor. For spherical particles, $P(\theta)$ is derived as ${ }^{14}$

$$
\begin{aligned}
P(\theta) & =\left[\frac{3}{x^{3}}(\sin x-x \cos x)\right]^{2}, \\
x & =k a
\end{aligned}
$$

where $a$ is the radius of the sphere. For randomly coiled polymers undergoing translational diffusion, the autocorrelation functions, both for the field and for the photocurrent, are given by expressions similar to those of spherical particles. However the form factor $P(\theta)$ of randomly coiled polymers is different and is given by ${ }^{15}$

$$
\begin{aligned}
P(\theta) & =\frac{2}{t^{2}}[\exp (-t)-(1-t)], \\
t & =\frac{\left\langle R^{2}\right\rangle k^{2}}{6}
\end{aligned}
$$

where $\left\langle R^{2}\right\rangle$ is the mean square end-to-end distance of the Gaussian coiled polymer. For rod-sphaped molecules, we have to consider rotational diffusion as well as translational diffusion. Cummins, et al. ${ }^{8}$ showed that the autocorrelation function of the photocurrent of a monodisperse 
rod-shaped molecule with the length $L$ is expressed by

$$
C^{\prime}(\tau)=\langle i\rangle^{2}\left[\frac{\sum_{l} N P_{l}(\theta)|A(M, 0)|^{2} \exp \left[-\left(D_{\mathrm{T}} k^{2}+l(l+1) D_{\mathrm{R}}\right) \tau\right]}{\sum_{l} N P_{l}(\theta)|A(M, 0)|^{2}}\right]^{2}
$$

where $D_{\mathrm{R}}$ is the rotational diffusion constant. The form factor of a rod-shaped molecule is given by

$$
P_{l}(\theta)=(2 l+1)\left[\frac{1}{h} \int \frac{\hat{J}_{l}(x)}{x} \mathrm{~d} x\right]^{2}, \quad h=\frac{L}{2} \quad \text { and } \quad \hat{J}_{l}(x)=\sqrt{\frac{\pi x}{2}} J_{l+1 / 2}(x)
$$

The autocorrelation function of a polydisperse rod-shaped molecule is similarly given by

$$
C(\tau)=\frac{\sum_{l} \int_{0}^{\infty} P_{l}(\theta)|A(M, 0)|^{2} f(M) \exp \left[-\left(D_{\mathrm{T}} k^{2}+l(l+1) D_{\mathrm{R}}\right) \tau\right] \mathrm{d} M}{\sum_{l} \int_{0}^{\infty} P_{l}(\theta)|A(M, 0)|^{2} f(M) \mathrm{d} M}
$$

The apparent molecular-weight distribution function for a polydisperse rod-shaped molecule is defined as

$$
W_{l}(M, \theta)=\frac{P_{l}(\theta)|A(M, 0)|^{2} f(M)}{\sum_{l} \int_{0}^{\infty} P_{l}(\theta)|A(M, 0)|^{2} f(M) \mathrm{d} M}
$$

We represent $C(\tau)$ by the summation over the components as

$$
C(\tau)=\sum_{l} C_{l}(\tau)
$$

Then we obtain the following Laplace transform:

$$
C_{l}(\tau)=\int_{0}^{\infty} W_{l}(M, \theta) \exp (-x \tau)\left[\frac{\mathrm{d}\left[D_{\mathrm{T}} k^{2}+l(l+1) D_{\mathrm{R}}\right]}{\mathrm{d} M}\right] \mathrm{d} x
$$

where $x=D_{\mathrm{T}}(M) k^{2}+l(l+1) D_{\mathrm{R}}(M)$.

This result can again be used to obtain the molecular-weight distribution function of a polydisperse solution of rod-shaped molecules.

\section{AVERAGE DIFFUSION CONSTANT OF POLYDISPERSE POLYMER SOLUTION}

One of the methods to obtain translational diffusion constant is the initial slope method. The initial slope of the photocurrent autocorrelation function is obtained as follows. For a monodisperse system, the translational diffusion constant is obtained from the slope of $\ln C(\tau)$ $\tau$ relation. For a polydisperse system, $\ln C(\tau)-$ $\tau$ relation is not always linear. We define the initial slope by

$$
\left.\frac{\mathrm{d} \ln C(\tau)}{\mathrm{d} \tau}\right|_{\tau \rightarrow 0}
$$

Differentiating the logarithum of eq 15 , we obtain the initial slope as

$$
\left.\frac{\mathrm{d} \ln C(\tau)}{\mathrm{d} \tau}\right|_{\tau \rightarrow 0}=-k^{2}\left\langle D_{\mathrm{T}}(M)\right\rangle
$$

$$
\begin{aligned}
& \text { where } \\
& \begin{aligned}
\left\langle D_{\mathrm{T}}(M)\right\rangle & =\int_{0}^{\infty} D_{\mathrm{T}}(M) W(M) \mathrm{d} M \\
& =\frac{\int_{0}^{\infty} D_{\mathrm{T}}(M) f(M)|A(M, \theta)|^{2} \mathrm{~d} M}{\int_{0}^{\infty} f(M)|A(M, \theta)|^{2} \mathrm{~d} M}
\end{aligned}
\end{aligned}
$$

As has been shown above, the normalized intensity $P(\theta)$ is defined by

$$
P(\theta)=\frac{|A(M, \theta)|^{2}}{|A(M, 0)|^{2}}
$$

Average translational diffusion constant $\left\langle D_{\mathrm{T}}(M)\right\rangle$ is then given by

$$
\left\langle D_{\mathrm{T}}(M)\right\rangle=\frac{\int D_{\mathrm{T}}(M) M^{2} f(M) P(\theta) \mathrm{d} M}{\int M^{2} f(M) P(\theta) \mathrm{d} M}
$$




\section{T. TANAKA}

In eq $30,|A(M, 0)|^{2}$ is taken to be

$$
|A(M, 0)|^{2} \propto M^{2}
$$

For the case $k=0$, i.e., $\theta=0$,

$$
\begin{aligned}
\left\langle D_{\mathrm{T}}(M)\right\rangle & =\frac{\int D_{\mathrm{T}}(M) M^{2} f(M) \mathrm{d} M}{\int M^{2} f(M) \mathrm{d} M} \\
& =\left\langle D_{\mathrm{T}}(M)\right\rangle_{z}
\end{aligned}
$$

where $\left\langle D_{\mathrm{T}}(M)\right\rangle_{z}$ denotes the $z$-average of $D_{\mathrm{T}}(M)$.

Expanding eq 19 in the range $x \ll 1$, we get

$$
P(\theta) \approx 1-\frac{x^{2}}{5}
$$

Substituting eq 33 into eq 30 , we obtain

$$
\begin{aligned}
\left\langle D_{\mathrm{T}}(M)\right\rangle= & \left\langle D_{\mathrm{T}}(M)\right\rangle_{z} \\
& +\frac{k^{2}}{5}\left\langle a^{2}(M)_{z}\left(\left\langle D_{\mathrm{T}}(M)\right\rangle-\left\langle D_{\mathrm{T}}(M)\right\rangle_{\mathrm{sp}}\right)\right.
\end{aligned}
$$

where $\left\langle a^{2}(M)\right\rangle_{z}$ denotes the $z$-average of $a^{2}(M)$ and $\left\langle D_{\mathrm{T}}(M)\right\rangle_{\mathrm{sp}}$ is defined by

$$
\left\langle D_{\mathrm{T}}(M)_{\mathrm{sp}}=\frac{\int D_{\mathrm{T}}(M) f(M) M^{2} a^{2}(M) \mathrm{d} M}{\int f(M) M^{2} a^{2}(M) \mathrm{d} M}\right.
$$

As has been shown in eq $34,\left\langle D_{\mathrm{T}}(M)\right\rangle$ depends on the scattering angle. This effect is interpreted as follows. Diffusion constant of a solution depends on the size of the molecule. Diffusion constant of larger particles gives a lower value than that of smaller particles. The distribution of molecular size influences the scattered intensity as has been explained in the previous section. There is a region of scattering angles in which the contribution of large molecules is predominant. There also exists a region in which this effect is not so important. In the scattering angle region where larger molecules give a predominant contribution, the observed average diffusion constant becomes smaller. In this way, a scattering angle dependence of the average diffusion constant will be observed for a polydisperse system.

Calculating in a way similar to the case of spheres, $\left\langle D_{\mathrm{T}}(M)\right\rangle$ of a polydisperse Gaussian coiled molecule is given by

$$
\begin{aligned}
\left\langle D_{\mathrm{T}}(M)\right\rangle= & \left\langle D_{\mathrm{T}}(M)\right\rangle_{z} \\
& +\frac{k^{2}}{18}\left\langle R^{2}(M)\right\rangle_{z}\left[\left\langle D_{\mathrm{T}}(M)\right\rangle-\left\langle D_{\mathrm{T}}(M)\right\rangle_{g}\right]
\end{aligned}
$$$$
(x \ll 1)
$$

where $\left\langle D_{\mathrm{T}}(M)\right\rangle_{g}$ is defined as

$$
\left\langle D_{\mathrm{T}}(M)\right\rangle_{g}=\frac{\int D_{\mathrm{T}}(M) f(M) M^{2}\left\langle R^{2}(M)\right\rangle \mathrm{d} M}{\int f(M) M^{2}\left\langle R^{2}(M)\right\rangle \mathrm{d} M}
$$

and

$$
\begin{aligned}
&\left\langle D_{\mathrm{T}}(M)\right\rangle=\left\langle D_{\mathrm{T}}(M)\right\rangle_{z} \\
&+\frac{k^{2}}{6}\left\langle R^{2}(M\rangle_{n}\left(\left\langle D_{\mathrm{T}}(M)\right\rangle-\left\langle D_{\mathrm{T}}(M)\right\rangle_{w}\right)\right. \\
&(x \gg 1)
\end{aligned}
$$

where $\left\langle D_{\mathrm{T}}(M)\right\rangle_{n}$ the $\left\langle R^{2}(M)\right\rangle_{n}$ denote the number average of $D_{\mathrm{T}}(M)$ and $R^{2}(M)$ respectively and $\left\langle D_{\mathrm{T}}(M)\right\rangle_{w}$ is weight average $D_{\mathrm{T}}(M)$.

In the case of rod-like scatterers, the situation is a little more complicated, because both the translational and rotational diffusion constants should be considered. When we treat only the translational part and neglect the rotational part, namely, if we put $l=0$ in eq 22 , the normalized intensity is given by

$$
P_{0}(\theta)=\left[\frac{1}{h} \int_{0}^{h} \frac{\sin x}{x} \mathrm{~d} x\right]^{2}
$$

eq 39 is expanded in the range of $h \ll 1$ as

$$
\begin{aligned}
P(\theta) & \approx\left[\frac{1}{h}\left(h-\frac{h^{3}}{3 ! 3}\right)\right]^{2} \\
& \approx 1-\frac{1}{9} h^{2}
\end{aligned}
$$

We then obtain an approximate expression for the averaged translational diffusion constant of rod-like scatterers:

$$
\begin{aligned}
\left\langle D_{\mathrm{T}}(M)\right\rangle= & \langle D(M)\rangle_{z} \\
& +\frac{k^{2}}{36}\left\langle L^{2}(M)\right\rangle_{z}\left[\left\langle D_{\mathrm{T}}(M)\right\rangle-\left\langle D_{\mathrm{T}}(M\rangle_{\mathrm{rod}}\right]\right.
\end{aligned}
$$

where $\left\langle D_{\mathrm{T}}(M)\right\rangle_{\mathrm{rod}}$ is defined as

$$
\left\langle D_{\mathrm{T}}(M)\right\rangle_{\mathrm{rod}}=\frac{\int D_{\mathrm{T}}(M) f(M) M^{2} L^{2}(M) \mathrm{d} M}{\int f(M) M^{2} L^{2}(M) \mathrm{d} M}
$$




\section{APPLICATION TO SEVERAL SYSTEMS}

\section{Blend of Spheres}

We apply the preceding theory to the quasielastic light scattering of several model systems. We consider a solution which consists of a blend of the same kind of monodisperse spherical molecules. The spectral distribution of a blend system is given by

$$
I(\omega)=\left(\frac{1}{\pi}\right) \sum_{i} \frac{w_{i} D_{\mathrm{T}}\left(M_{i}\right) k^{2} M_{i}^{2} P(\theta)}{\left(\omega-\omega_{0}\right)^{2}+\left(D_{\mathrm{T}}\left(M_{i}\right) k^{2}\right)^{2}}
$$

where $w_{i}$ is the weight fraction of the $i$-th molecule with the molecular weight $M_{i}$. The autocorrelation function of the photocurrent and the average diffusion constant of a blend system are respectively given as follows:

$$
\begin{gathered}
C(\tau)=\frac{\sum_{i} w_{i} M_{i}{ }^{2} P(\theta) \exp \left(-D_{\mathrm{T}}\left(M_{i}\right) k^{2} \tau\right)}{\sum_{i} w_{i} M_{i}{ }^{2} P(\theta)} \\
D_{\mathrm{T}}(M)=\frac{\sum_{i} w_{i} D_{\mathrm{T}}\left(M_{i}\right) M_{i}{ }^{2} P(\theta)}{\sum_{i} w_{i} M_{i}{ }^{2} P(\theta)}
\end{gathered}
$$

We assume that the molecular weight of the sphere is proportional to the third power of its radius $\left(M \propto a^{3}\right)$ and use the Stokes relation $D_{\mathrm{T}}=$ $C / a(C$, constant; $a$, radius). Numerical calculations have been performed for the blend of spheres with diameter $d_{1}=0.1 \mu$, molecular weight

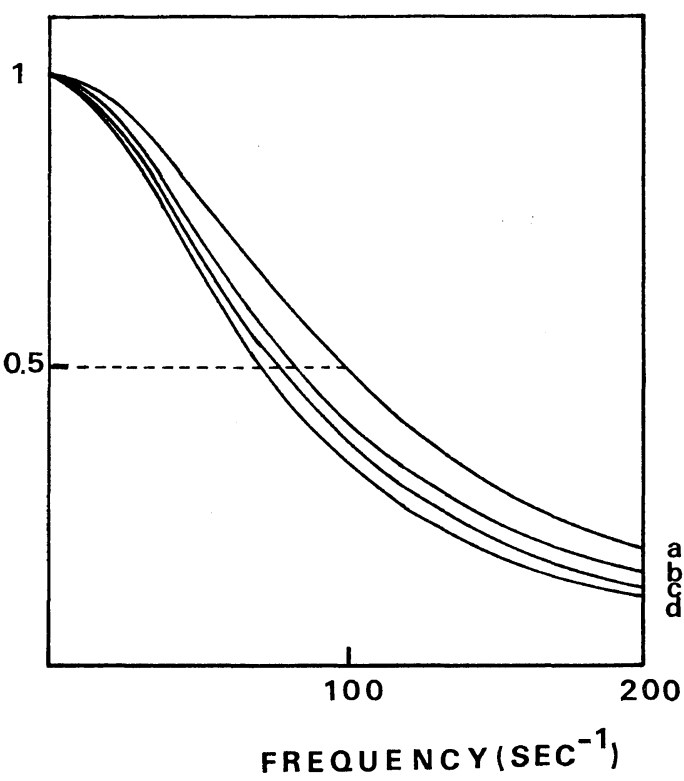

(a)

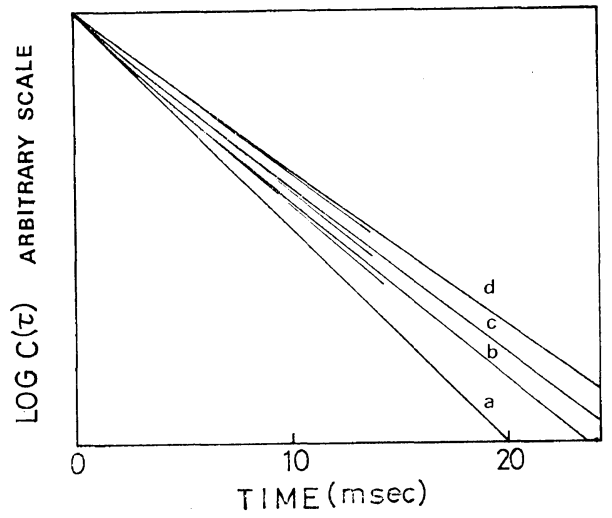

(b)

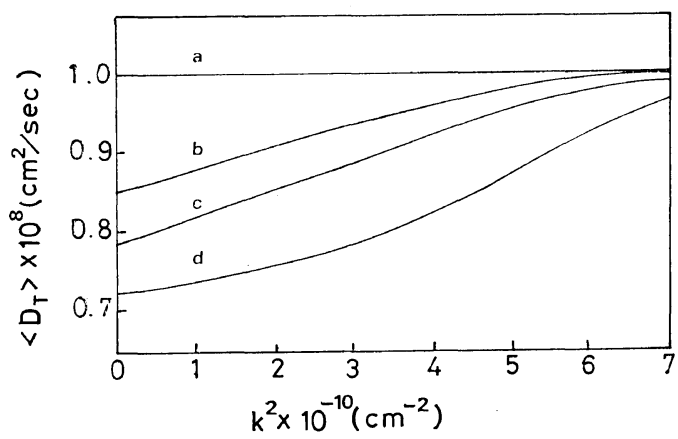

(c)

Figure 1. (a) spectral distribution at $k=1 \times 10^{5}$ $\left(\mathrm{cm}^{-1}\right)$, (b) autocorrelation function of the photocurrent at $k=1 \times 10^{5}\left(\mathrm{~cm}^{-1}\right)$, (c) average diffusion constant for a blend of spheres of $0.1 \mu \mathrm{m}$ and 0.3 $\mu \mathrm{m}$ in diameter. The weight fraction of $0.3 \mu \mathrm{m}$ spheres (in diam.) is; (a) 0 , (b) 0.1 , (c) 0.2 , (d) 0.5 .

$M_{w}=10^{9}$ and of spheres with diameter $d_{2}=3 d_{1}$. Figure 1a gives plots of the spectra for the weight fraction of the large sphere $w_{2}=0,0.1$, 0.2 , and 0.5 at $k=1 \times 10^{5}\left(\mathrm{~cm}^{-1}\right)$. The half-widths of the spectra of the blend systems are lower than that of the monodisperse system. Therefore the average diffusion constant of the blend system shows a lower value than that of the monodisperse system with the same mean molecular weight. Figure $1 \mathrm{~b}$ gives plots of the autocorrelation function of the photocurrent of the blend systems. They show deviation from a straight line in the plot of $\ln C(\tau)-\tau$. This method is more appropriate to estimate the effect of polydispersity as compared with the method of examining the spectral distribution. 
Plots of the average diffusion constants of the blend systems are shown in Figure 1c. The average diffusion constant becomes smaller, the higher the weight fraction of large molecular and/or the smaller the scattering angle.

\section{Continuous Molecular Weight Distribution of Sphere}

We consider a system which consists of the same kind of spheres with a continuous distribution of the molecular weight. We use the Schulz-Zimm distribution function for $f(M):{ }^{16}$

$$
f(M)=(1 / z !)\left[\frac{z+1}{M_{w}}\right]^{z+1} M^{z} \exp \left[-\frac{(z+1) M}{M_{w}}\right]
$$

where $M_{w}$ denotes the weight average of the molecular weight and $z$ is the polydispesity parameter. Substituting eq 45 into eq 7 , we have the spectral distribution of the system. Numerical calculations have been performed for spheres with $d=0.2 \mu$ and $M_{w}=10^{9}$. The Stokes relation and the relation $M \propto a^{3}$ are also assumed. Results for polydispersity parameter $z=1,3$, and $\infty$ (monodisperse) at $k=1 \times 10^{5}\left(\mathrm{~cm}^{-1}\right)$ are presented in Figure 2a. The average diffusion constant of a polydisperse system is expected to give a lower value than that of the monodisperse system with the same mean molecular weight. The autocorrelation function of the photocurrent and the

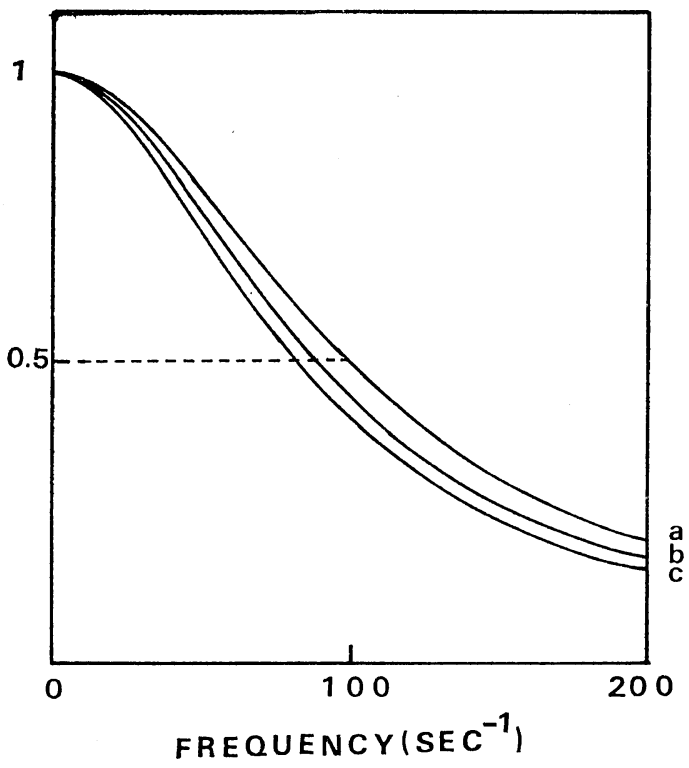

(a)

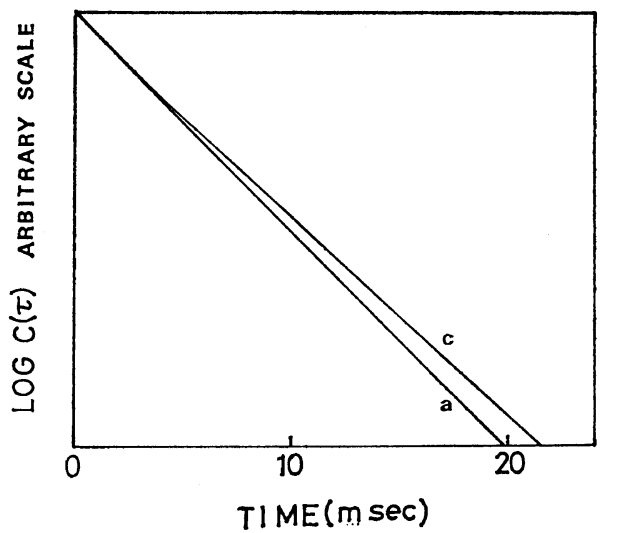

(b)

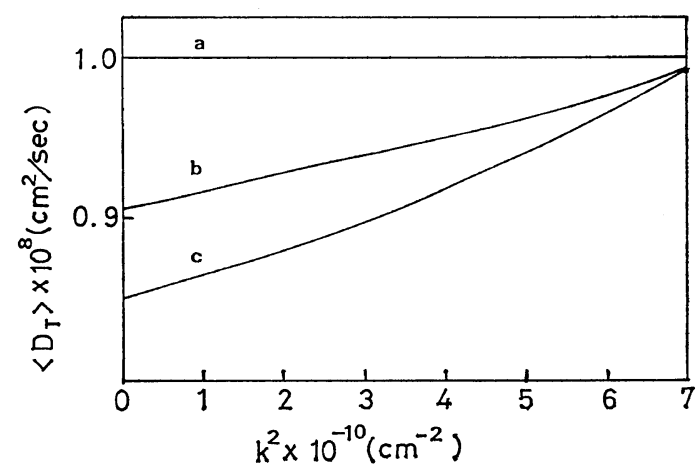

(c)

Figure 2. Same plots as in Figure 1 for a polydisperse sphere with Schulz-Zimm's distribution of the molecular weight. The polydispersity parameter is; (a) $z=\infty$ (monodisperse), (b) $z=3$, (c) $z=1$.

average diffusion constant of the polydisperse system are similarly obtained by eq 13 and eq 29. Results are respectively shown in Figure $2 b$ and Figure 2c. Slight deviation from linearity in the $\ln C(\tau)-\tau$ relation is noted at large values of the polydispersity. There, the scattering angle dependence of the average diffusion constant is also remarkable.

\section{Gaussian Coiled Polymer}

We consider a system which consists of Gaussian coiled polymers with the Schulz-Zimm distribution function of the molecular weight. The spectral distribution and the autocorrelation fnnction of the photocurrent are similarily obtained. The mean-square end-to-end distance $\left\langle R^{2}\right\rangle$ of the polymer is proportional to the molecular weight of the molecule and the diffusion 
Light Scattering from Polydisperse Polymer

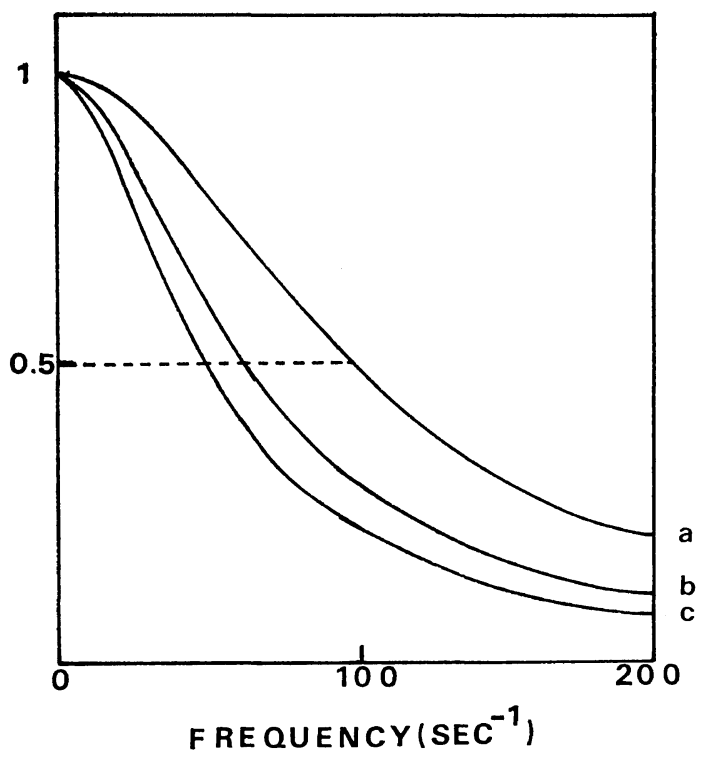

(a)

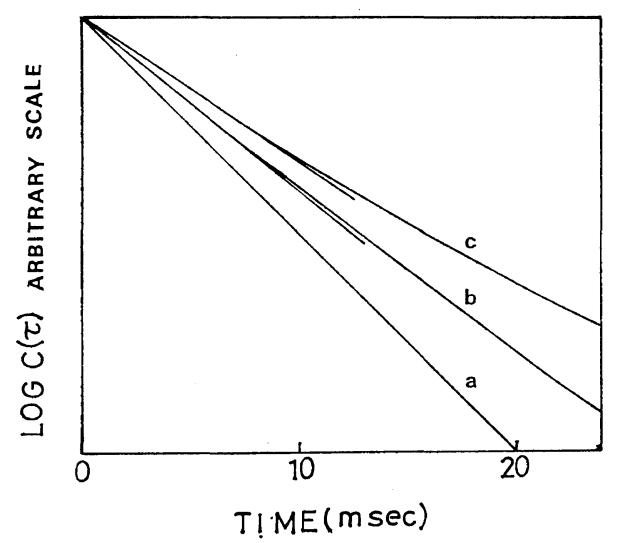

(b)

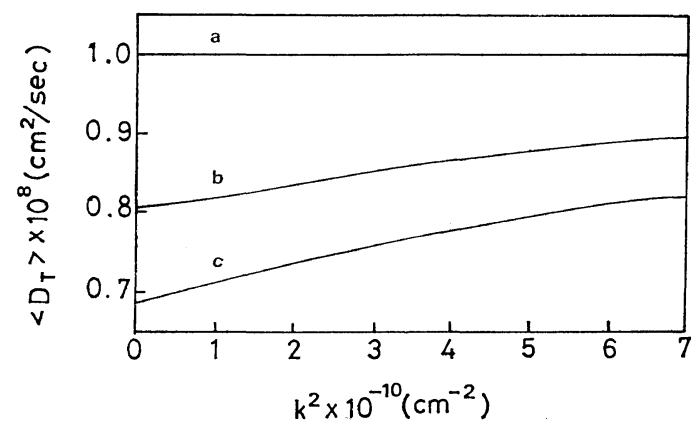

(c)

Figure 3. Same plots as in Figure 1 for a polydisperse Gaussian coiled polymer with SchulzZimm's distribution of the molecular weight for $\alpha=1$. The polydispersity parameter is; (a) $z=\infty$, (b) $z=3$, (c) $z=1$.

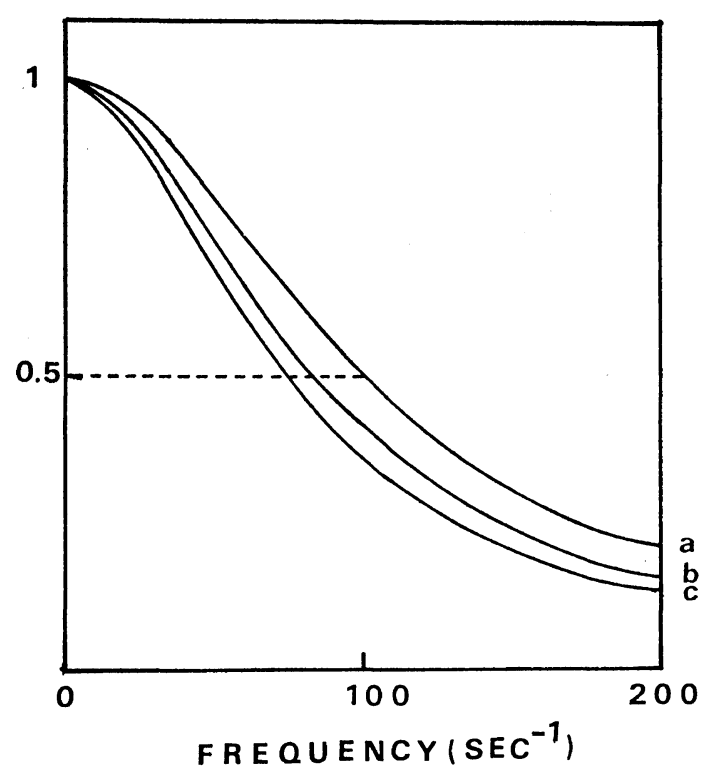

(a)

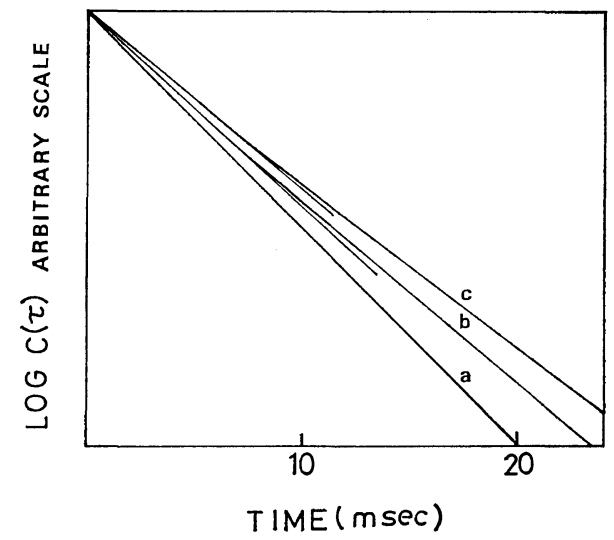

(b)

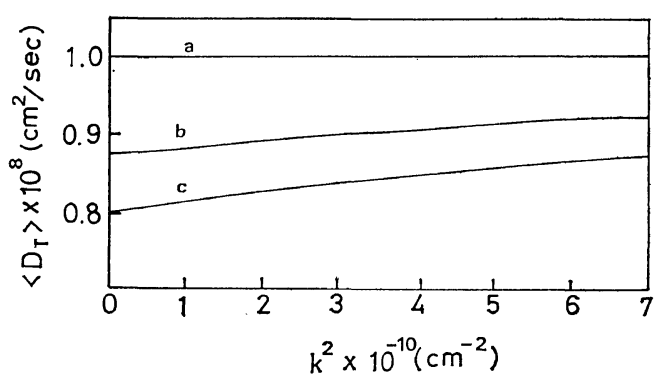

(c)

Figure 4. Same plots as in Figure 1 for a polydisperse Gaussian coiled polymer with SchulzZimm's distribution of the molecular weight for $\alpha=0.5$. The polydispersity parameter is; (a) $z=\infty$, (b) $z=3$, (c) $z=1$. 


\section{T. TANAKA}

constant $D_{\mathrm{T}}$ is related to the molecular weight through the equation $D_{\mathrm{T}}=C M^{-\alpha}$ for Gaussian coiled polymers. The case of $\alpha=0.5$ corresponds to the so-called nondraining molecule and the case of $\alpha=1$ to the draining molecule. Numerical calculations have been performed for Gasussian coiled polymers with $\left\langle R^{2}\right\rangle=2000 \AA$ and $M_{w}=10^{8}$. Results of the spectra for $z=1,3$, and $\infty$ (monodisperse) at $k=1 \times 10^{5}\left(\mathrm{~cm}^{-1}\right)$ for $\alpha=1$ are presented in Figure 3a. Similar result for $\alpha=0.5$ is shown in Figure 4a. Results of the autocorrelation function of the photocurrent and the average diffusion constant of these systems are respectively shown in Figure $3 b$ and Figure $3 c$ for $\alpha=1$ and in Figure $4 \mathrm{~b}$ and Figure $4 \mathrm{c}$ for $\alpha=0.5$.

\section{RESULTS AND DISCUSSION}

Average diffusion constants calculated for several model systems in the previous section are listed in Table I. The average diffusion constant obtained by the half-width of the spectrum is different from the obtained directly from the autocorrelation function of the photocurrent. It is caused by the deviation from a Lorentzian form of the spectrum due to the polydispersity. The rigorous analysis of the polydispersity effect on the spectrum form is treated by Koppel. ${ }^{12}$ According to his theory, the spectrum of the light scattered from a polydisperse solution is given by

$$
\begin{aligned}
I(\omega)= & \frac{A}{\pi} \frac{\left\langle D_{\mathrm{T}}\right\rangle k^{2}}{\left(\omega-\omega_{0}\right)^{2}+\left(\left\langle D_{\mathrm{T}}\right\rangle k^{2}\right)^{2}}\left[1+\frac{\left(\left\langle D_{\mathrm{T}}\right\rangle k^{2}\right)^{2}-3\left(\omega-\omega_{0}\right)^{2}}{\left(\left(\omega-\omega_{0}\right)^{2}+\left(\left\langle D_{\mathrm{T}}\right\rangle k^{2}\right)^{2}\right)^{2}} M_{2}\right. \\
& \left.+\frac{\left(\left\langle D_{\mathrm{T}}\right\rangle k^{2}\right)^{4}-6\left(\left\langle D_{\mathrm{T}}\right\rangle k^{2}\right)^{2}\left(\omega-\omega_{0}\right)^{2}+\left(\omega-\omega_{0}\right)^{4}}{\left(\left\langle D_{\mathrm{T}}\right\rangle k^{2}\right)\left(\left(\left\langle D_{\mathrm{T}}\right\rangle k^{2}\right)^{2}+\left(\omega-\omega_{0}\right)^{2}\right)^{3}} M_{3}+\cdots\right]
\end{aligned}
$$

where

$$
M_{m}=\left\langle\left(D_{\mathrm{T}} k^{2}-\left\langle D_{\mathrm{T}}\right\rangle k^{2}\right)^{m}\right\rangle .
$$

The first approximation of eq 47 is

where

$$
I(\omega)=\frac{A}{\pi} \frac{\left\langle D_{\mathrm{T}}\right\rangle k^{2}}{\left(\omega-\omega_{0}\right)^{2}+\left(\left\langle D_{\mathrm{T}}\right\rangle k^{2}\right)^{2}}\left[1+\frac{\left(\left\langle D_{\mathrm{T}}\right\rangle k^{2}\right)^{2}-3\left(\omega-\omega_{0}\right)^{2}}{\left.\left(\left\langle D_{\mathrm{T}}\right\rangle k^{2}\right)^{2}+\left(\omega-\omega_{0}\right)^{2}\right)^{2}}\left(\left\langle D_{\mathrm{T}}{ }^{2}\right\rangle k^{4}-\left(\left\langle D_{\mathrm{T}}\right\rangle k^{2}\right)^{2}\right)\right]
$$

$$
\begin{aligned}
\left\langle D_{\mathrm{T}}(M)^{2}\right\rangle & =\int_{0}^{\infty} D_{\mathrm{T}}(M)^{2} W(M) \mathrm{d} M \\
& =\frac{\int D_{\mathrm{T}}(M)^{2} f(M)|A(M, \theta)|^{2} \mathrm{~d} M}{\int f(M)^{2}|A(M, \theta)|^{2} \mathrm{~d} M}
\end{aligned}
$$

We confirm numerically that the spectrum obtained from Koppel's equation (eq 47) with the values of $\left\langle D_{\mathrm{T}}(M)\right\rangle$ and $\left\langle D_{\mathrm{T}}(M)^{2}\right\rangle$ obtained by our theory agrees with those plotted in Figures
$1 a, 2 a, 3 a$, and $4 a$. It is pointed out that the

\begin{tabular}{|c|c|c|c|c|c|c|c|c|c|c|}
\hline & \multirow{3}{*}{$\begin{array}{l}\text { Monodisperse } \\
\text { system }\end{array}$} & \multirow{2}{*}{\multicolumn{3}{|c|}{$\begin{array}{c}\text { Blend of spheres } \\
\begin{array}{l}\text { Weight fraction of } 0.3 \\
\mu \mathrm{m} \text { (in dia.) sphere }\end{array}\end{array}$}} & \multirow{2}{*}{\multicolumn{2}{|c|}{$\begin{array}{c}\begin{array}{c}\text { Polydisperse } \\
\text { sphere }\end{array} \\
\end{array}$}} & \multicolumn{4}{|c|}{ Polydisperse polymer } \\
\hline & & & & & & & \multicolumn{2}{|c|}{$\alpha=0.5$} & \multicolumn{2}{|c|}{$\alpha=1$} \\
\hline & & 0.1 & & $\begin{array}{l}\text { here } \\
0.5\end{array}$ & $z=1$ & $z=3$ & $z=1$ & $\overline{z=3}$ & $z=1$ & $z=3$ \\
\hline & 1 & 0.84 & 0.78 & 0.72 & 0.83 & 0.89 & 0.74 & 0.83 & 0.50 & 0.63 \\
\hline $\begin{array}{l}\text { Autocorrelarion- } \\
\text { function method }\end{array}$ & 1 & 0.88 & 0.82 & 0.74 & 0.87 & 0.92 & 0.81 & 0.88 & 0.71 & 0.82 \\
\hline
\end{tabular}
polydispersity causes deviation from a Lorentzian ditribution and average diffusion constants obtained from the half-width at the half-maximum

Table I. Average diffusion constant of several model systems obtained both from the spectral method and from the autocorrelation function method at $k=1 \times 10^{5}\left(\mathrm{~cm}^{-1}\right){ }^{\mathrm{a}}$

a Unit, $10^{-8} \mathrm{~cm}^{2} / \mathrm{sec}$. 
of the spectrum do not give exact values for polydisperse solution. Although our theory is equivalent to that of Koppel in some special cases, our theory is more directly applicable to the data of the autocorrelation function of the photocurrent. It also explains the scattering angle dependence of the average diffusion constant of the polydisperse system.

\section{CONCLUSIONS}

The autocorrelation function formulation for the quasielastic light scattering from a polydispese polymer solution has been proposed. The spectrum and the autocorrelation function of the photocurrent of light scattered from a polydisperse solution have been discussed. The autocorrelation function of the photocurrent is given by a Laplace transform of the apparent molecular-weight distribution function which depends on the scattering intensity. The effect of polydispersity is clearly found in the autocorrelation function of the photocurrent. The relation of $\ln C(\tau)-\tau$ shows deviation from a straight line in the polydisperse system. On the other hand, deviation from a Lorentzian form of the spectrum caused by the polydispersity is much less clear. The average diffusion constant of the polydisperse system $\left\langle D_{\mathrm{T}}(M)\right\rangle$ is obtained both from the spectral distribution and the autocorrelation function. It shows a lower value than that of the monodisperse solution with the same mean molecular weight. The average diffusion constant at $\theta=0$ is the $z$-average of the diffusion constant. The scattering angle dependence of $\left\langle D_{\mathrm{T}}(M)\right\rangle$ is also shown. The average diffusion constant of the polydisperse system calculated from the autocorrelation function of the photocurrent given an exact value, whereas that calculated from the half-width of the spectrum involves some approximations.

Acknowledgments. The author wishes to express her thanks to the late Prof. M. Yamamoto of Tokyo Metropolitan University and Prof. K. Nishikawa of Hiroshima University for their kind discussions. She is also indepted to Prof. M. H. Birnboim of Rensselaer Polytechnique Institute and Dr. H. Tanaka of Mitsui Petrochemical Co. Ltd., for calling her attention to the experiments of the light scattering from the polymer solution.

\section{REFERENCES}

1. R. Pecora, J. Chem. Phys., 40, 1604 (1964).

2. R. Pecora, ibid., 43, 1562 (1965).

3. R. Pecora, ibid., 48, 4126 (1968).

4. R. Pecora, ibid., 49, 1032, 1036 (1968).

5. R. Pecora, Macromolecules, 2, 31 (1969).

6. B. J. Berne and R. Pecora, J. Chem. Phys. 50, 783 (1969).

7. B. J. Berne and R. Pecora, ibid., 51, 475 (1969).

8. H. Z. Cummins, F. D. Carlson, T. J. Herbert, and G. Woods, Biophys. J., 8, A95 (1968); 9, 518 (1969).

9. Y. Tagami and R. Pecora, J. Chem. Phys., 51, 3293, 3298 (1969).

10. D. S. Thompson, ibid., 75, 789 (1971).

11. J. E. Frederick, T. F. Reed and O. Kramer, Macromolecules, 4, 242 (1971).

12. D. E. Koppel, J. Chem. Phys. 57, 4814 (1972).

13. G. Mie, Ann. Phys., 25, 377 (1908).

14. L. Rayleigh, Proc. Roy. Soc. Ser A, (London) 90, 219 (1914).

15. B. H. Zimm, R. S. Stein, and P. Doty, Polym. Bull., 1, 90 (1945).

16. B. H. Zimm, J. Chem. Phys., 16, 1099 (1948). 